\title{
Sensitization of Midbrain Dopamine Neuron Reactivity Promotes the Pursuit of Amphetamine
}

\author{
Paul Vezina,, ${ }^{1,2}$ Daniel S. Lorrain, ${ }^{3}$ Gretchen M. Arnold, ${ }^{1}$ Jennifer D. Austin, ${ }^{1}$ and Nobuyoshi Suto ${ }^{1}$ \\ ${ }^{1}$ Department of Psychiatry and ${ }^{2}$ Committee on Neurobiology, The University of Chicago, Chicago, Illinois 60637, and \\ 3Merck Research Labs, San Diego, La Jolla, California 92037-4641
}

Stimulant drugs such as amphetamine are readily selfadministered by humans and laboratory animals by virtue of their actions on dopamine (DA) neurons of the midbrain. Repeated exposure to this drug systemically or exclusively in the cell body region of these neurons in the ventral tegmental area (VTA) leads to long-lasting changes in dopaminergic function that can be assessed by increased locomotor activity and enhanced DA overflow in the nucleus accumbens (NAcc) after re-exposure to the drug. Three experiments were conducted to evaluate the possibility that this enduring sensitized reactivity underlies compulsive drug self-administration. In all experiments, rats were pre-exposed to amphetamine and, starting $10 \mathrm{~d}$ later, their intravenous self-administration of the drug was assessed. In the first experiment, rats previously exposed to amphetamine systemically or exclusively in the VTA subsequently worked harder than untreated animals to obtain the drug when the work required to obtain successive infusions

Psychomotor stimulants such as amphetamine share with a number of abused drugs the ability to increase extracellular levels of dopamine (DA) in the nucleus accumbens (NAcc), the major subcortical terminal field of midbrain DA neurons originating in the ventral tegmental area (VTA). In rodents, this action is associated with increased locomotion and exploration and with the capacity of these drugs to support self-administration (Wise and Bozarth, 1987; Koob, 1992).

Repeated exposure to amphetamine (Kalivas and Stewart, 1991), as well as to nonpharmacological stressors (Piazza and Le Moal, 1996), leads to a long-term enhancement (weeks to months in the rat) in the ability of this drug to increase locomotion and to elevate extracellular levels of DA in the NAcc. Amphetamine is known to act in the region of the A10 DA cell bodies in the VTA, and not in DA terminal regions such as the NAcc or the prefrontal cortex, to initiate the development of these effects (Kalivas and Weber, 1988; Hooks et al., 1992; Perugini and Vezina, 1994; Cador et al., 1995; Vezina, 1996). These findings together with the considerable evidence linking the mesoaccumbens DA system not only to the locomotion produced but also to the selfadministration supported by psychomotor stimulant drugs (Wise and Bozarth, 1987) has, in the past decade, directed attention to

\footnotetext{
Received Nov. 29, 2001; revised March 1, 2002; accepted March 5, 2002.

This work was supported by United States Public Health Service (USPHS) Grants DA09397 and DA09860 (P.V.). D.S.L. was supported by USPHS Grant T32-DA-07255.

Correspondence should be addressed to Paul Vezina, Department of Psychiatry, The University of Chicago, 5841 South Maryland Avenue, MC 3077, Chicago, IL 60637. E-mail: pvezina@yoda.bsd.uchicago.edu.

Copyright (ㄷ) 2002 Society for Neuroscience $0270-6474 / 02 / 224654-09 \$ 15.00 / 0$
}

was increased progressively. In the second experiment, this progressively increasing workload was found to decrease the magnitude of amphetamine-induced DA overflow observed with successive infusions until responding ceased. Rats previously exposed to amphetamine were more resistant to this decline and more apt to maintain responding. Finally, in experiment three, a noncontingent priming injection of the drug produced a greater NAcc DA response and a greater parallel increase in lever pressing in drug compared with saline preexposed rats. Together, these results demonstrate a direct relation between drug-induced sensitization of midbrain dopamine neuron reactivity and the excessive pursuit and selfadministration of an abused substance.

Key words: sensitization; dopamine neuron reactivity; amphetamine self-administration; ventral tegmental area; nucleus accumbens; progressive ratio; drug pre-exposure; priming

the possibility that previous exposure to these drugs may potentially affect individuals' subsequent predisposition to pursue drug taking. Several reports have supported this possibility. In a manner paralleling sensitization of the locomotor and NAcc DA responses to amphetamine and cocaine, previous exposure to these drugs has been shown to enhance the acquisition and expression of conditioned place preferences (Lett, 1989; Shippenberg and Heidbreder, 1995) and to produce a long-lasting enhancement in animals' predisposition to pursue and selfadminister them (Piazza et al., 1989, 1990; Horger et al., 1990, 1992; Valadez and Schenk, 1994; Pierre and Vezina, 1997; Mendrek et al., 1998; Lorrain et al., 2000). However, although these findings are consistent with the view that sensitization of midbrain DA neuron reactivity underlies compulsively maintained drug self-administration (Robinson and Berridge, 1993), direct evidence for this idea continues to be lacking. Using a procedure that requires rats to press a lever a progressively increasing number of times to obtain successive infusions of the drug (Lorrain et al., 2000), three experiments assessed the impact of this sensitization on drug-taking behavior. It was first determined whether previous exposure to amphetamine in the VTA, the site where this drug is known to act to produce locomotor and NAcc DA sensitization, also enhances the self-administration of the drug (Experiment 1). In vivo microdialysis was then used to determine whether the differential responding for drug observed in rats previously exposed to systemic amphetamine or saline parallels these animals' differential NAcc DA response to the drug, whether actively (Experiment 2) or passively (Experiment 3) administered. The results obtained indicate a direct relation between drug- 
induced sensitization of midbrain DA neuron reactivity and the excessive pursuit and self-administration of the drug.

\section{MATERIALS AND METHODS}

\section{Subjects}

Male Long-Evans rats (Harlan Sprague Dawley, Madison, WI), weighing 250-300 gm on arrival, were used. They were housed individually in a $12 \mathrm{hr}$ light/dark reverse cycle room with food and water available ad libitum. Animals were allowed to acclimate to these conditions for 3-4 d before the start of any procedures. All testing was conducted during the dark period of the light cycle. Rats were fitted with an intravenous catheter in the right external jugular vein. Some rats were also implanted bilaterally with guide cannulas aimed at the VTA [anteroposterior (AP) -5.6 , mediolateral (ML) \pm 0.6 , dorsoventral (DV) -8.9] or the NAcc (AP $+2.4, \mathrm{ML} \pm 1.5, \mathrm{DV}-7.5 \mathrm{~mm}$ from skull and bregma). Cannulas were angled at $16^{\circ}$ (VTA) or $10^{\circ}$ (NAcc) to the vertical and positioned 1 $\mathrm{mm}$ above the final injection site. Obturators were inserted into the guide cannulas after surgery. Rats used in the microdialysis experiments were fitted with an intravenous catheter and implanted unilaterally with a guide cannula aimed at the NAcc (DV -6.5 to -8.5 from skull; Paxinos and Watson, 1986). This cannula was angled at $10^{\circ}$ to the vertical and positioned $5 \mathrm{~mm}$ above the ventralmost aspect of the NAcc. All intracranial implants were made before and intravenous catheters fitted after the pre-exposure injections (see Design and procedure). Rats were given a 7-10 d recovery period after the intracranial implants. All surgical procedures were conducted under sterile conditions with the animal under deep anesthesia (ketamine, $100 \mathrm{mg} / \mathrm{kg}$, i.p.; xylazine, $6 \mathrm{mg} / \mathrm{kg}$, i.p.) as previously described in reports from this laboratory (intravenous catheters, Pierre and Vezina, 1997; intracranial implants, Perugini and Vezina, 1994; microdialysis implants, Jolly and Vezina, 1996, Vezina, 1996) and, together with all remaining experimental procedures, according to an approved Institutional Animal Care and Use Committee protocol.

\section{Design and procedure}

All experiments consisted of a drug pre-exposure phase and, starting $10 \mathrm{~d}$ later, testing for amphetamine self-administration. Intravenous catheters were fitted at least 5-7 d before the beginning of self-administration testing.

Experiment 1. Rats in different groups were pre-exposed to amphetamine or saline either into the VTA, the NAcc, or intraperitoneally, as described below. Unless stated otherwise, the concentration of amphetamine used throughout the subsequent intravenous self-administration phase was $200 \mu \mathrm{g} / \mathrm{kg}$ per infusion. To establish a dose-effect curve, rats in separate groups were pre-exposed to amphetamine intraperitoneally and subsequently tested with one of five self-administered doses of amphetamine $(50,70,100,200$, or $300 \mu \mathrm{g} / \mathrm{kg} /$ infusion $)$ under the progressive ratio (PR) schedule. Rats tested with $50-100 \mu \mathrm{g} / \mathrm{kg}$ per inf usion under the PR schedule first self-administered $100 \mu \mathrm{g} / \mathrm{kg}$ per infusion under the fixed ratio (FR) schedules. Rats tested with $200-300 \mu \mathrm{g} / \mathrm{kg}$ per infusion under the PR schedule first self-administered $200 \mu \mathrm{g} / \mathrm{kg}$ per inf usion under the FR schedules.

Experiment 2. Rats were pre-exposed to intraperitoneal amphetamine or saline and tested for their self-administration of $200 \mu \mathrm{g} / \mathrm{kg}$ per infusion of amphetamine as outlined below. Microdialysis was used to estimate extracellular levels of DA in the NAcc in animals selfadministering amphetamine on days 4 to 5 of PR testing. To confirm the amphetamine dependence of the self-administration and NAcc DA responses observed during testing, separate rats in a first control group (no drug control group) were treated as those above but, on the microdialysis test day, received no infusions after depression of the lever. Additional rats in a second control group (FR control group) were tested to permit the assessment of the specific impact of the increasing workload imposed by the PR schedule of reinforcement on the ability of amphetamine to maintain self-administration and NAcc DA responding to successive infusions of the drug. These control animals received no pre-exposure injections and were trained to emit a constant amount of work (five presses: FR5) to receive successive amphetamine infusions. They were tested daily on the FR5 schedule until microdialysis testing after 3-4 d of self-administration on this schedule. Microdialysis probes were lowered into the NAcc on the day before microdialysis testing, and rats were kept in dialysis chambers for the next 18-20 hr. After the collection of four baseline samples, rats were moved to the self-administration chambers, and an additional three baseline samples were collected before the levers were inserted, and more samples were collected. Rats tested under the PR schedule were tested for an additional $240 \mathrm{~min}$, the no drug control rats for an additional $100 \mathrm{~min}$, and the FR control rats for an additional $180 \mathrm{~min}$. Dialysate samples were collected every $5 \mathrm{~min}$.

Experiment 3. As in Experiment 2, rats were pre-exposed to intraperitoneal amphetamine or saline and tested for their self-administration of $200 \mu \mathrm{g} / \mathrm{kg}$ per infusion of amphetamine as outlined below. In this case, however, microdialysis was used on days 4 to 5 of PR testing to estimate extracellular levels of DA in the NAcc before and after a single noncontingent priming injection of amphetamine $(1.0 \mathrm{mg} / \mathrm{kg}$, i.p.). The priming injection was made as the levers were introduced into the chambers. Subsequent pressing on the levers had no intravenous consequences. Rats were tested for $180 \mathrm{~min}$ after the priming injection. Dialysate samples were collected every $20 \mathrm{~min}$.

\section{Pre-exposure injections}

Pre-exposure injections (intraperitoneal: $5 \times 1.5 \mathrm{mg} / \mathrm{kg}$ amphetamine or $1.0 \mathrm{ml} / \mathrm{kg}$ saline; intracranial: $4 \times 2.5 \mu \mathrm{g} / 0.5 \mu \mathrm{l}$ per side of amphetamine or $0.5 \mu \mathrm{l} / \mathrm{side}$ of saline) were administered by the experimenter, one injection given every third day (Perugini and Vezina, 1994; Vezina, 1996). During this phase, some rats in experiment 1 were placed in activity monitoring boxes immediately after the first and last injection, and their locomotor activity was measured for $2 \mathrm{hr}$. In all remaining cases, animals were transported to an experimental room, administered their respective injections, and returned to their home cages. Intracranial microinjections were made in the freely moving rat. Injection cannulas connected to $1 \mu \mathrm{l}$ Hamilton syringes via PE-20 tubing were inserted to a depth of $1 \mathrm{~mm}$ below the guide cannula tips. Injections were made in a volume of $0.5 \mu \mathrm{l} / \mathrm{side}$ during a $30 \mathrm{sec}$ period. The injection cannulas were removed, and the obturators were replaced $60 \mathrm{sec}$ later. All doses refer to the weight of the salt of $S(+)$-amphetamine sulfate (Sigma, St. Louis, $\mathrm{MO})$.

\section{Locomotor activity}

A bank of 12 activity boxes was used to measure locomotion in some rats during the pre-exposure phase in experiment 1 . Each box $(22 \times 43 \times 33$ $\mathrm{cm}$ ) was constructed of opaque plastic (rear and two side walls), a Plexiglas front-hinged door, and a tubular stainless steel ceiling and floor. Two photocells, positioned $3.5 \mathrm{~cm}$ above the floor and spaced evenly along the longitudinal axis of each box, estimated locomotion. Two additional photocells, positioned on the side walls $16.5 \mathrm{~cm}$ above the floor and $5 \mathrm{~cm}$ from the front and back walls, estimated rearing. Separate interruptions of photocell beams were detected and recorded via an electrical interface by a computer situated in an adjacent room using locally developed software. The activity chambers were kept in a room illuminated dimly with red light.

\section{Self-administration}

Twelve test chambers were used for amphetamine self-administration testing. Each chamber measured $22 \times 22 \times 33 \mathrm{~cm}$, was constructed of the same materials described above, and was enclosed in a plastic box that shielded animals from extraneous disturbances. White noise was supplied in each box by a ventilating fan. A single retractable response lever $(5 \mathrm{~cm}$ above the floor) and a stimulus light $(13.5 \mathrm{~cm}$ above the lever) were positioned on the left side wall. Each chamber was equipped with a liquid swivel to allow free movement of the animal in the chamber and delivery of drug after depression of the lever. Each animal was connected to the swivel via a steel-spring tether attached to the animal by screwing its captive collar onto the threaded portion of a custom designed L-shaped Plastics One (Roanoke, VA) cannula (20 gauge) secured to the animal's skull. Infusions were delivered by Razel infusion pumps (model A.E.; Razel Scientific Inc.). Lever presses and drug infusions were recorded and controlled via an electrical interface by a computer using locally developed software.

Starting $10 \mathrm{~d}$ after the drug pre-exposure phase, rats were required to self-administer 10 infusions of amphetamine intravenously in a $4 \mathrm{hr}$ session on first an FR1 and then an FR2 schedule of reinforcement. Sessions lasted either until rats self-administered the 10 inf usions or until the $4 \mathrm{hr}$ had elapsed. Animals satisfying each criterion within $5 \mathrm{~d}$ were then tested on a PR schedule of reinforcement in each of five or six daily $4 \mathrm{hr}$ sessions. Number of responses required to obtain successive infusions under the PR schedule was determined by ROUND $(5 \cdot$ EXP$(0.25 \cdot$ infusion number $)-5)$ to produce the following sequence of required lever presses: 1, 3, 6, 9, 12, 17, 24, 32, 42, 56, 73, 95, 124, 161, 208, etc. The PR sessions lasted $4 \mathrm{hr}$ or until $1 \mathrm{hr}$ elapsed without a drug 
inf usion. The final ratio attained with a PR schedule has been defined as the subject's break point (Richardson and Roberts, 1996; Lorrain et al., 2000).

Rats in one of the control groups in experiment 2 (FR control group) received no pre-exposure injections and were trained to emit five lever presses (FR5) to receive successive amphetamine infusions. Before testing on the FR5 schedule of reinforcement, they were required to self-administer 10 infusions of amphetamine intravenously in a $4 \mathrm{hr}$ session on first an FR1, an FR2, and then an FR3 schedule of reinforcement. All of these animals satisfied each criterion within $5 \mathrm{~d}$.

Of the 231 animals tested, 11 (seven amphetamine and four saline pre-exposed) failed to satisfy the FR criteria and were excluded. After completion of the experiment, the remaining rats were administered a lethal dose of Nembutal via their jugular catheter. Animals that did not lose muscle tone within 5-10 sec and expire promptly were ruled to have nonpatent catheters and were excluded (11: again, seven amphetamine and four saline pre-exposed).

\section{In vivo microdialysis and chromatography}

Eight chambers $(38 \times 32 \times 34 \mathrm{~cm})$ constructed of the same materials described above and enclosed in a plastic box were used to house animals after microdialysis probe insertion and before moving them to the self-administration chambers. Animals were connected via a steel-spring tether to a liquid swivel and collection vial positioned outside the plastic shell. Concentric microdialysis probes were constructed in the laboratory and perfused with a modified Ringer's dialysate at $1.5 \mu \mathrm{l} / \mathrm{min}$ (Jolly and Vezina, 1996; Vezina, 1996).

In self-administering rats, samples were collected at 5 min intervals and frozen for later assessment of DA by an HPLC system using electrochemical detection (EC) and consisting of a $300 \mu \mathrm{m}$ inner diameter (i.d.) $15 \mathrm{~cm}$ column packed with $3 \mu \mathrm{m}$ C-18 BDS particles, an Antec micro electrochemical detector (using a $22 \mathrm{nl}$ volume microflow cell with a glassy carbon working electrode maintaining an applied potential of +0.7 $\mathrm{V}$ relative to the $\mathrm{Ag}-\mathrm{AgCl}$ reference electrode), and a $0.09 \mathrm{M}$ sodium acetate mobile phase containing $35 \mathrm{~mm}$ citric acid (Hull et al., 1995). In rats tested after a single priming injection, samples were collected at $20 \mathrm{~min}$ intervals and processed immediately with an HPLC-EC system consisting of a $4.6 \mathrm{~mm}$ i.d. $10 \mathrm{~cm}$ column packed with $3 \mu \mathrm{m}$ C-18 ODS particles, a Coulochem detector (conditioning cell oxidizing at $+300 \mathrm{mV}$; analytical cell electrodes set to -50 and $-350 \mathrm{mV}$ ), and a $0.04 \mathrm{M}$ sodium acetate mobile phase (Jolly and Vezina, 1996; Vezina, 1996).

\section{Histology}

After verification of catheter patency and expiration of the animals, the brains of those rats that had been fitted with intracranial implants to permit drug microinjection or microdialysis were then removed and stored in $30 \%$ sucrose-formalin solution. Forty micrometer coronal sections were mounted on gelatin-coated slides and stained with cresyl violet for verification of cannula tip and microdialysis probe placements. Nine rats were pre-exposed to saline injections in sites outside the VTA. As was found with rats pre-exposed to amphetamine in sites outside the VTA (Fig. 1C), these animals also failed to distinguish themselves from VTA saline pre-exposed rats during self-administration testing.

\section{Data analyses}

The pre-exposure locomotor data in experiment 1 were analyzed with between-within ANOVA with drug (two levels: amphetamine and saline) as the between factor and days (two levels: first and last injection day) as the within factor. The self-administration data collected in the different experiments were analyzed either with $t$ tests for independent samples (days to criterion under the FR schedules, number of infusions obtained averaged over six PR test days and duration of responding and number of infusions obtained or earned in a single PR test session in saline vs amphetamine pre-exposed rats), one-way between ANOVA (number of infusions obtained averaged over six PR test days) with pre-exposure condition as the between factor (three VTA pre-exposure levels) as well as two-way between ANOVA (number of infusions obtained averaged over five PR test days) with pre-exposure (two levels: amphetamine and saline) and self-administered dose of amphetamine (five levels) as the between factors in the dose-response experiment. The NAcc DA data collected in experiments 2 and 3 were analyzed either with $t$ tests for independent samples (peak levels attained in saline vs amphetamine pre-exposed rats) as well as one-way within ANOVA (DA levels at and after a single amphetamine infusion) with time (three levels) as the within factor. Post hoc Scheffé comparisons were made according to Kirk
(1968). For all self-administration data obtained with the PR schedule, the actual data analyzed were the number of infusions obtained or earned rather than the number of presses emitted or the final ratios obtained because the latter were derived, by definition, from an exponential function (Richardson and Roberts, 1996).

\section{RESULTS}

\section{Experiment 1. Rats previously exposed to amphetamine intraperitoneally or into the VTA, but not into the NAcc, will subsequently work more to obtain the drug}

As expected, when compared with saline after the first and last pre-exposure injection, amphetamine consistently produced substantial and statistically significant increases in horizontal locomotion and rearing when administered intraperitoneally $\left(F_{(1,17)}=37.3\right.$ and $10.7 ; p<0.001$ and 0.005 , respectively) and into the NAcc $\left(F_{(1,17)}=15.8\right.$ and $\left.17.8 ; p<0.001\right)$, but not when administered into the VTA $\left(F_{(1,17)}=0.0004\right.$ and 0.27 ; NS). No significant effects of day or group $\times$ day interactions were detected. Although substantial increases in locomotion are observed acutely when amphetamine is administered systemically or into the NAcc, large increases in locomotion from the first to the last day of pre-exposure are rarely observed with these injections in such designs. With systemically administered amphetamine, this most likely reflects the progressive recruitment of competing stereotypic behaviors that also show sensitization (Stewart and Vezina, 1987; Crombag et al., 1999). In such cases, as in those where amphetamine is administered into the VTA and does not increase locomotion acutely (present findings; Kalivas and Weber, 1988; Perugini and Vezina, 1994), evidence that sensitization has developed is reliably obtained by subsequently challenging animals either with lower systemic doses of the drug or by administering it via a different route. The results of such tests have consistently shown that rats previously exposed to amphetamine intraperitoneally or in the VTA, but not the NAcc, show enhanced locomotor responding to a subsequent challenge injection of the drug when compared with animals previously exposed to saline (see introductory remarks). Similarly, in the present experiments, enhanced responding resulting from these amphetamine relative to saline pre-exposure injections was subsequently assessed by testing rats for their intravenous self-administration of the drug.

Interestingly, when initially required to self-administer up to 10 infusions per session of a high dose of the drug $(200 \mu \mathrm{g} / \mathrm{kg}$ per infusion) on first an FR1 and then an FR2 schedule, amphetamine and saline pre-exposed rats were indistinguishable and readily acquired the lever press response. In three separate experiments (one each with intraperitoneal, VTA, or NAcc preexposure), amphetamine pre-exposed rats acquired responding in $3.0 \pm 0.4,2.4 \pm 0.2$, and $2.8 \pm 0.3$ sessions and saline pre-exposed rats in $3.3 \pm 0.5,3.0 \pm 0.5$, and $3.5 \pm 0.6$. $t$ tests comparing saline and amphetamine pre-exposed animals in each experiment revealed $p$ values $>0.05$. In contrast, when animals were then required to lever press more and more to obtain each successive infusion under a PR schedule of reinforcement, those previously exposed to amphetamine intraperitoneally pressed threefold to fivefold more often, and as a result obtained more infusions of the drug $\left(t_{17}=2.2 ; p<0.025\right)$ and did so over several days of testing (Fig. $1 A$ ). This enhancement was observed over a range of selfadministered drug doses. It can be seen in Figure $1 B$ that under the PR schedule, higher doses of the self-administered drug supported more work. In rats pre-exposed to amphetamine, this effect was magnified within the limits afforded by the PR schedule 
A. I.P. Pre-exposure

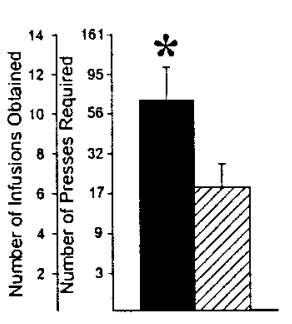

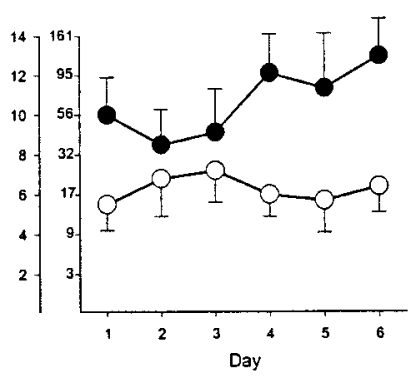

B. Dose-effect curve

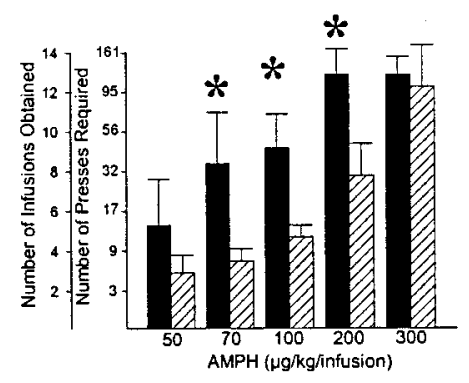

C. VTA Pre-exposure
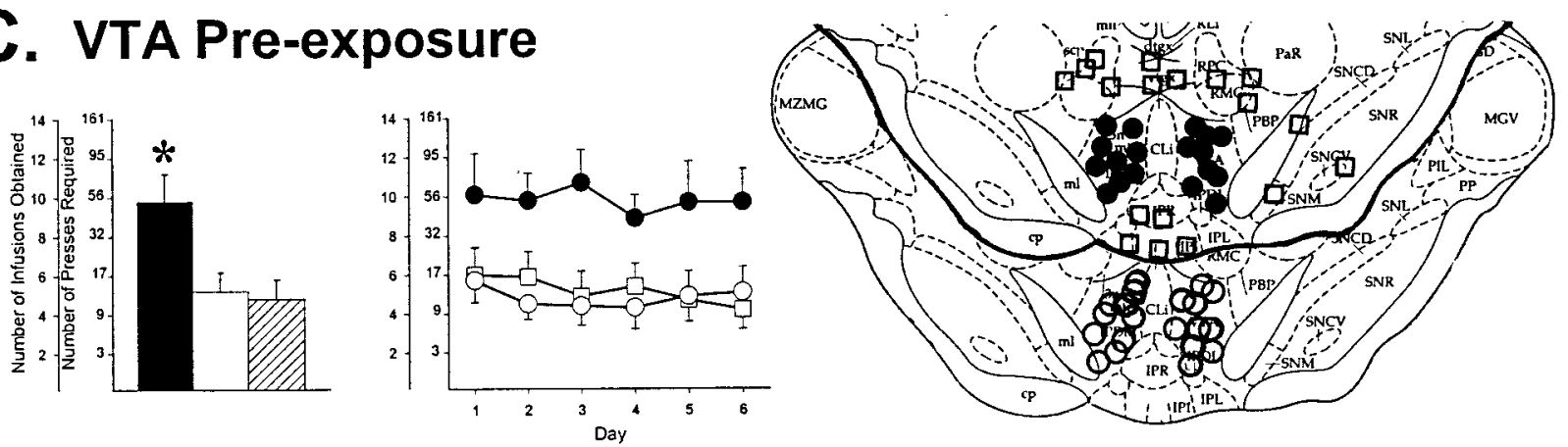

D. N.Acc. Pre-exposure
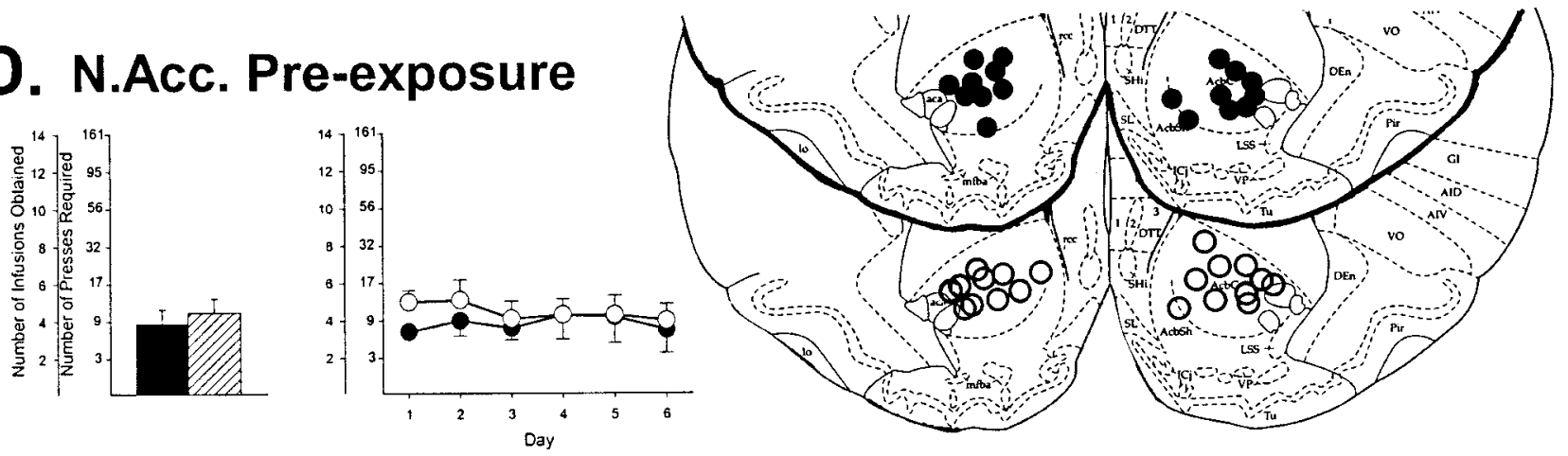

\section{Pre-exposure}
/O amphetamine
EIIT/O saline
amphetamine
outside VTA

Figure 1. Rats previously exposed to amphetamine intraperitoneally or into the VTA, but not into the NAcc, will subsequently work more to obtain the drug. In all cases, data are shown as mean ( \pm SEM) number of infusions obtained. The number of presses required to obtain the successive inf usions is also shown. $A$, Rats pre-exposed to amphetamine or saline intraperitoneally were tested for their self-administration of the $200 \mu \mathrm{g} / \mathrm{kg}$ per inf usion dose of the drug under a PR schedule. Bar graphs were derived from means of the values obtained for each subject on each of the six PR test days. These are shown to the right as group means. ${ }^{*} p<0.05$ as revealed by $t$ test. $n=8-11$ per group. $B$, Rats pre-exposed to amphetamine or saline intraperitoneally were tested for their self-administration of one of five doses of the drug. Bars were derived from means of the values obtained for each subject on each of five PR test days. * $p$ values $<0.05-0.025$ versus saline pre-exposed rats, as revealed by post hoc tests following ANOVA. $n=5-12$ per group. $C$, Rats pre-exposed to VTA amphetamine, VTA saline, or amphetamine in sites adjacent to the VTA were tested as in $A$. Injection cannula tip placements in the mesencephalon of rats in the different groups are shown at the far right. Overlaid line drawings (all adapted from Paxinos and Watson, 1986) each depict the caudal surface of a coronal section extending -5.2 to $-6.0 \mathrm{~mm}$ from bregma. ${ }^{*} p<0.01$ versus saline pre-exposed rats as revealed by $p o s t$ hoc tests after ANOVA. $n=9-11$ per group. $D$, Rats pre-exposed to NAcc amphetamine or saline were tested as in $A$. Injection cannula tip placements are illustrated as in $C$. Overlaid line drawings each depict the caudal surface of a coronal section extending +2.2 to $+3.0 \mathrm{~mm}$ from bregma. $n=9-10$ per group.

used and the amount of drug the animals could endure ( $p$ values $<0.05-0.025$ as revealed by post hoc tests after ANOVA). Concentrations in excess of $300 \mu \mathrm{g} / \mathrm{kg}$ per infusion produced erratic behavior and, as a result, did not support higher break points (data not shown); at these concentrations, all animals ceased to respond early in the session and, in most cases, exhibited signs of motor impairment and intense stereotypy. The ANOVA conducted on the data obtained with the $50-300 \mu \mathrm{g} / \mathrm{kg}$ 

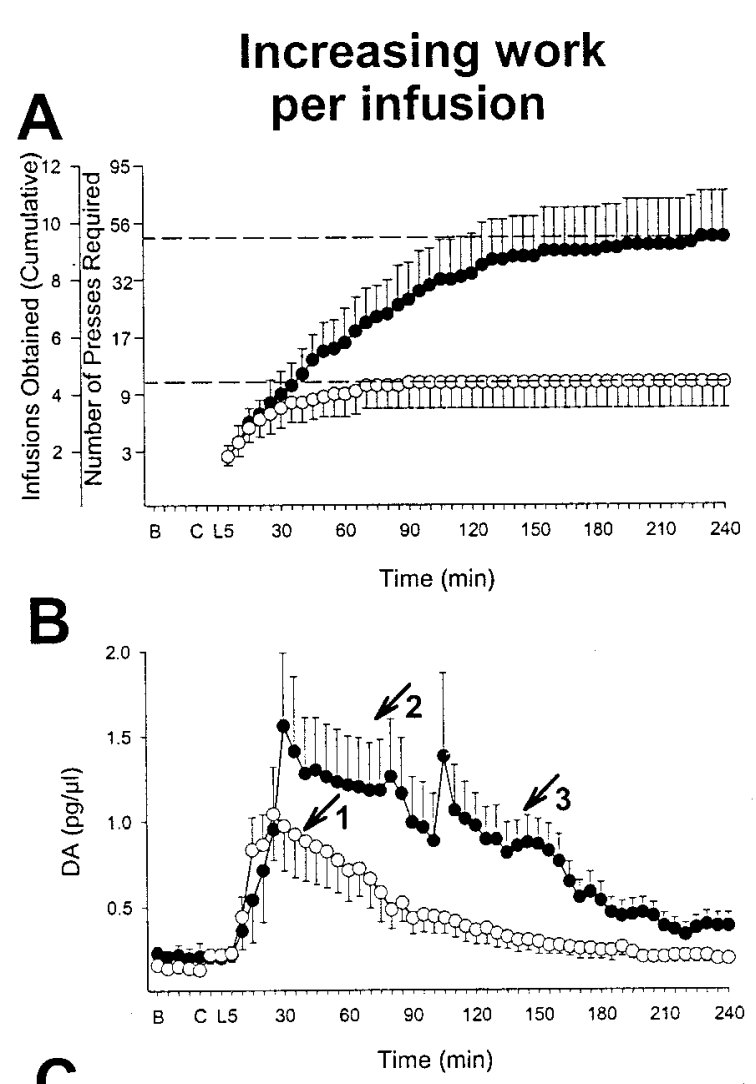

C

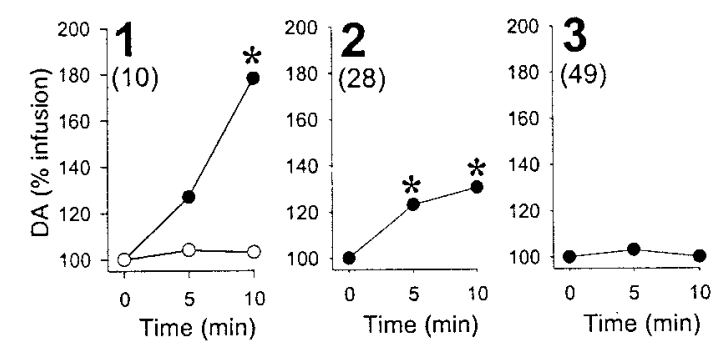

D

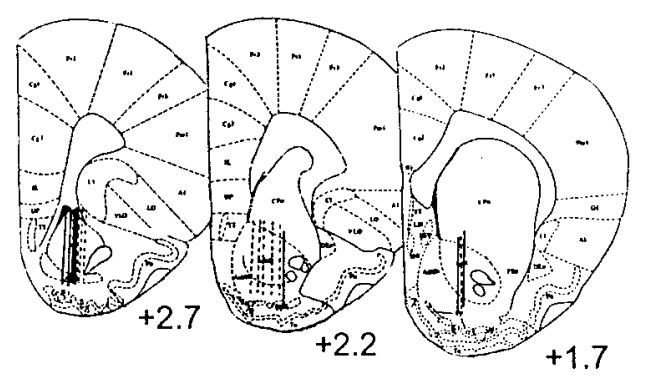

amphetamine pre-exposed $\bigcirc$ saline pre-exposed

Figure 2. Rats previously exposed to amphetamine maintain responding and a significant drug-induced increase in NAcc DA for a longer period of time when self-administering the drug under a PR schedule requiring progressively increasing work to obtain successive infusions. $A$, Cumulative record of infusions obtained on the fourth to the fifth day of PR testing. Amphetamine pre-exposed rats obtained significantly more infusions than saline pre-exposed rats (dashed lines: $9.5 \pm 1.6$ vs $4.4 \pm 0.9 ; p<$ 0.005). $B$, Baseline. $C$, Rats transferred to self-administration chamber. $L$, Lever inserted into chamber. $B$, Extracellular concentrations of DA in the NAcc before and during amphetamine self-administration during PR testing for rats described above. Data are shown as mean picograms per microliter $( \pm$ SEM) plotted over time. Dialysate samples were collected per infusion concentrations revealed significant effects of dose $\left(F_{(4,64)}=8.6 ; p<0.001\right)$ and pre-exposure $\left(F_{(1,64)}=10.3 ; p<\right.$ $0.002)$. Remarkably, similar effects were observed in those rats previously exposed to amphetamine in the VTA (Fig. 1C) $(p<$ 0.01 as revealed by post hoc tests after ANOVA showing a significant effect of pre-exposure, $\left.F_{(2,27)}=6.0 ; p<0.007\right)$, but not in those exposed to amphetamine in the NAcc (in spite of the fact such injections elicit dramatic increases in locomotion acutely; see above) or in sites surrounding the VTA (Fig. 1C,D).

\section{Experiment 2. Under conditions of progressively increasing workload, rats previously exposed to amphetamine maintain drug-induced increases in NAcc DA and responding for drug for a longer period of time than saline pre-exposed rats}

To assess the relation between the enhancement in drug selfadministration observed above and the enhanced NAcc DA overflow (Kalivas and Stewart, 1991; Vezina, 1996) that is also observed in animals previously exposed to amphetamine, rats in additional groups were prepared with intravenous catheters and unilaterally implanted guide cannulas aimed at the NAcc to permit in vivo microdialysis testing. After intraperitoneal preexposure injections of either amphetamine or saline and amphetamine self-administration training, extracellular levels of DA in the NAcc were estimated in self-administering rats on day 4-5 of testing on the PR schedule. The behavior of the animals during these sessions was characterized by an initial loading period in which rats in both groups similarly and rapidly self-administered the drug (Fig. $2 A$ ). This led to a precipitous rise in DA levels that peaked within $30 \mathrm{~min}(1.6 \pm 0.4 \mathrm{in}$ amphetamine and $1.0 \pm 0.3$ $\mathrm{pg} / \mu \mathrm{l}$ in saline pre-exposed rats; $p>0.05)$. After this period, DA levels remained 4- to 6.7-fold higher than levels observed before the animals began self-administering the drug and only returned to baseline after responding ceased (Fig. 2B, arrows 1, 3). Again, rats previously exposed to amphetamine emitted more lever presses, continued to respond significantly longer (almost $2 \mathrm{hr} ; t_{17}=3.4 ; p<0.0025$ ) and obtained significantly more infusions (more than twofold; $t_{17}=2.9 ; p<0.005$ ) than saline pre-exposed rats. This difference can be accounted for by the fact that, in amphetamine pre-exposed rats, the individual amphetamine infusions continued to elicit a significant increase in NAcc DA (up to $78 \%$ increase in overflow) for a longer period of time during which the work required to obtain

$\leftarrow$

every $5 \mathrm{~min}$. Numbered arrows indicate the group mean time of 1 , the last infusion $(4.4 \pm 0.9)$ obtained by saline pre-exposed rats, and the infusion marking when amphetamine pre-exposed rats had obtained 50\% (4.5 \pm 0.9) of the infusions they would obtain in the session, 2, the infusion marking when amphetamine pre-exposed rats had obtained 75\% (7.5 \pm 1.2) of their infusions, and 3 , the last infusion $(9.5 \pm 1.6)$ obtained by amphetamine pre-exposed rats. Amphetamine pre-exposed rats continued to respond significantly longer than saline pre-exposed rats $(p<$ $0.005)$. $C$, DA concentrations at $(0 \mathrm{~min})$ and after $(5$ and $10 \mathrm{~min})$ the infusion obtained at times 1,2 , and 3 in $B$. Data are plotted as a percentage of levels observed at the time of the individual infusion. Amphetamine, compared with saline, pre-exposed rats resisted the decline in the ability of the drug infusions to maintain a NAcc DA response. Numbers in parentheses indicate the increasing group mean number of lever presses required to obtain the respective infusions. ${ }^{*} p$ values $<$ 0.01-0.001 versus levels at the time of infusion as revealed by post hoc tests after ANOVA. D, Line drawings of coronal sections showing the location of the active portion of the microdialysis probes in the NAcc (solid lines, amphetamine pre-exposed; dashed lines, saline pre-exposed). Numbers indicate millimeters from bregma. $n=8-11$ per group. 


\section{Constant work per infusion}

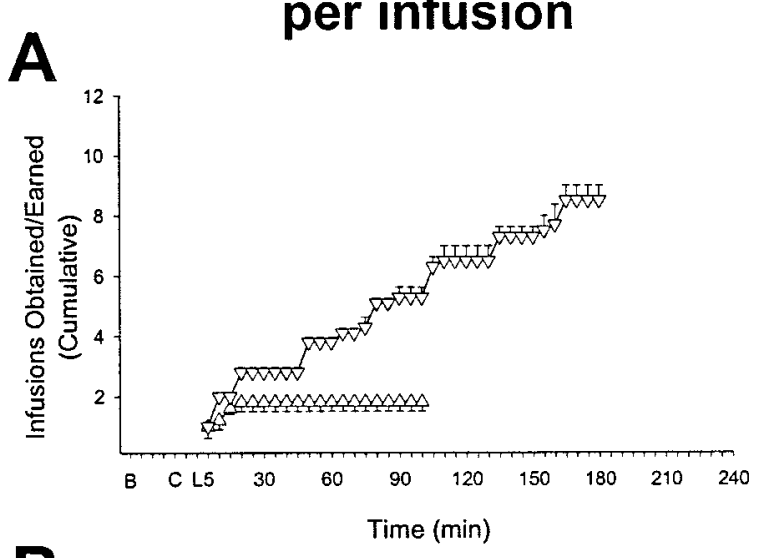

B
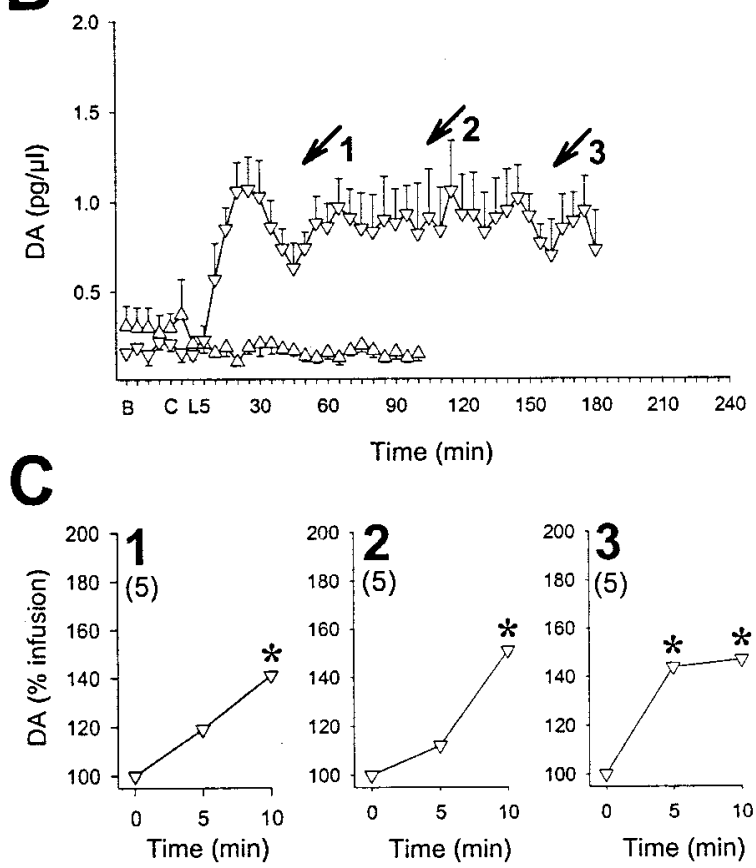

Time (min)
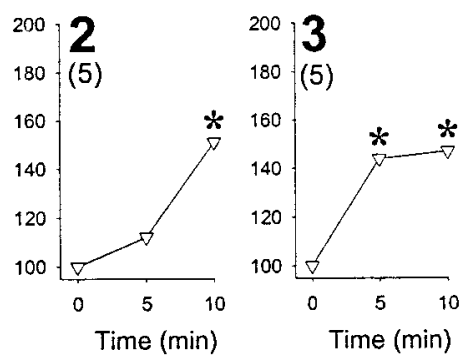

D

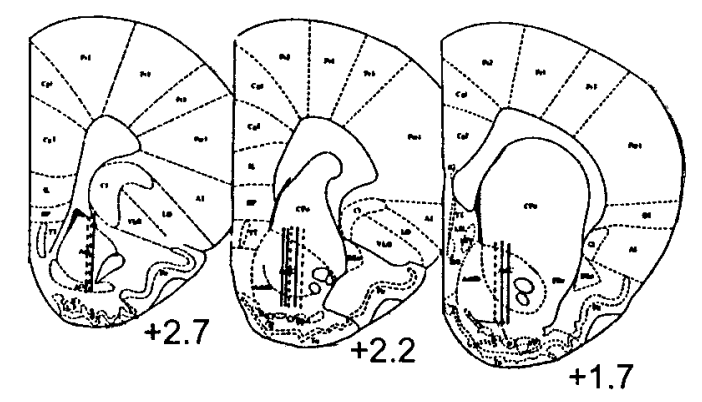

$\nabla$ FR control group

$\triangle$ No Drug control group

Figure 3. Rats for which the work required to obtain infusions is kept constant continue to press and show significant drug-induced increases in NAcc DA through to the last infusion of the session. Data are illustrated as in Figure 2 but for the FR and no drug control groups. FR control rats were trained to emit a constant amount of work (5 presses; FR5) to receive successive amphetamine infusions, were tested for $3 \mathrm{hr}$, and obtained $8.5 \pm 0.5$ infusions. Numbered arrows in $B$ indicate the group mean time of the infusion marking when these animals obtained $1,50 \%$ successive infusions increased progressively (Fig. 2C). Interestingly, the magnitude of the drug-induced DA overflow observed decreased as the amount of work required to obtain each injection increased. Amphetamine pre-exposed animals were resistant to this decline seen very early in the session in saline pre-exposed animals. In both groups, however, when the amphetamine inf usion was no longer accompanied by a significant increase in DA, responding ceased. It is noteworthy that at the time $(39.5 \pm 8.7 \mathrm{~min})$ of the last infusion $(4.4 \pm 0.9)$ in saline pre-exposed rats, amphetamine pre-exposed animals had only obtained $\sim 50 \%(4.5 \pm 0.9)$ of the total number of infusions $(9.5 \pm 1.6)$ they would self-administer in the session and, unlike the saline pre-exposed rats, continued to show a robust increase in NAcc DA overflow $(p<0.01)$ after the inf usion of amphetamine (Fig. $2 B$, arrow 1, C, plot 1$)\left(F_{(2,12)}=\right.$ $7.9 ; p<0.007$; one rat was omitted from this analysis because it self-administered an additional infusion during the $10 \mathrm{~min}$ post-infusion period analyzed and, as a result, showed inflated DA values). This significant increase in NAcc DA overflow continued to be in evidence, although less marked, after these animals had obtained $75 \%(7.5 \pm 1.2)$ of their infusions $(p$ values $<0.01-0.001)$ (Fig. $2 B$, arrow 2, C, plot 2) $\left(F_{(2,14)}=\right.$ $13.9 ; p<0.001)$. NAcc DA overflow was not significantly increased after the last self-administered infusion in either group (saline pre-exposed; Fig. $2 C$, plot $1 ; F_{(2,20)}=0.06, \mathrm{NS}$; amphetamine pre-exposed; Fig. $2 C$, plot $3 ; F_{(2,14)}=0.2$, NS).

These findings are in marked contrast to what was observed in control rats for which the work required to obtain infusions was kept constant (Fig. 3) (five presses for each infusion). These animals showed lever press responding that was relatively steady throughout the microdialysis test session (Fig. $3 A$ ). After the initial rise, DA levels remained 4.3- to 6.6-fold higher than baseline levels throughout the session (Fig. $3 B$ ). These animals continued to press, and significant increases in NAcc DA ( $p$ values $<$ $0.05-0.01)$ were observed in response to the individual amphetamine infusions through to the last inf usion of the session (as also reported by others; Ranaldi et al., 1999). The session was terminated by the experimenter at $180 \mathrm{~min}$. The ANOVA conducted on these data revealed significant increases in NAcc DA overflow after the individual infusions marking when these animals had obtained $50 \%$ (Fig. 3C, plot 1$)\left(F_{(2,10)}=4.8 ; p<0.05\right)$, $75 \%$ (Fig. $3 C$, plot 2$)\left(F_{(2,10)}=11.9 ; p<0.002\right)$ and $100 \%$ (last infusion) (Fig. 3C, plot 3) $\left(F_{(2,10)}=4.4 ; p<0.05\right)$ of the infusions they self-administered in the session. As shown in Figure 3, $A$ and $B$, rats in the no drug control group that received no inf usions after depression of the lever showed little pressing and no effect on NAcc DA.
$(4.2 \pm 0.2), 2,75 \%(6.3 \pm 0.3)$, and $3,100 \%$ (last infusion, $8.5 \pm 0.5)$ of their infusions. Numbers in parentheses in $C$ indicate the constant number of lever presses required to obtain the infusions self-administered at times 1,2 , and 3 in $B$. These animals maintained a significant increase in NAcc DA through to the last infusion of the session. ${ }^{*} p$ values $<$ 0.05-0.01 versus levels at the time of infusion as revealed by post hoc tests after ANOVA. No drug was delivered to no drug controls. These animals were tested for $100 \mathrm{~min}$ and showed little pressing and no effect on NAcc DA. Active portions of microdialysis probes are shown in $D$ (solid lines, FR control; dashed lines, no drug control). $n=5-6$ per group. 


\section{Experiment 3. Rats previously exposed to amphetamine show enhanced NAcc DA overflow and lever pressing in response to a single priming injection of the drug}

In experiments studying sensitization of amphetamine-induced locomotion and NAcc DA overflow, animals previously exposed to the drug or to saline are typically challenged at some later time with a noncontingent injection of the drug, and its effects are assessed. In self-administration paradigms, noncontingent injections have also been shown to reinstate drug-taking behaviors (Stewart et al., 1984; Shaham et al., 2000; Stewart, 2000). Given that these injections produce enhanced locomotor and NAcc DA effects in amphetamine pre-exposed animals, their effects on the generation of drug-taking behaviors was assessed. Rats in additional groups were trained as above, but on the microdialysis test day were given a single injection of the drug by the experimenter at the beginning of the session. As a result of this single noncontingent priming injection (Fig. 4, arrows), all animals showed increases in extracellular levels of DA in the NAcc that were accompanied in time by increased lever pressing, although presses were without consequence in this experiment. Rats previously exposed to amphetamine, however, showed higher levels of NAcc DA in response to the injection $\left(t_{14}=3.2 ; p<0.005\right)$ and made more presses on the lever $\left(t_{14}=1.8 ; p<0.05\right)$ than saline pre-exposed rats. The postinjection difference observed between groups in NAcc DA was also found to be significant when these data were expressed as changes from baseline levels observed before $\left(t_{14}=2.5 ; p<0.025\right)$ or after $\left(t_{14}=2.06, p<0.05\right)$ rats were transferred to the self-administration chambers. No significant differences between groups were observed in NAcc DA at either of these times before the priming injection.

\section{DISCUSSION}

In the present experiments, it was found that, in a manner similar to what has been observed with locomotion and NAcc DA overflow, rats previously exposed to amphetamine either intraperitoneally or in the VTA, but not in the NAcc, subsequently exhibited enhanced responding for the drug. These rats worked more than untreated animals to obtain the drug when the work required to obtain successive infusions was increased progressively. This progressively increasing workload was found to decrease the magnitude of amphetamine-induced DA overflow observed in the NAcc with successive infusions. When amphetamine no longer increased NAcc DA overflow, responding ceased. Rats previously exposed to amphetamine were more resistant to this decline and maintained responding for the drug for a longer period of time than saline pre-exposed animals. Finally, a noncontingent priming injection of amphetamine produced a greater NAcc DA response and a greater parallel increase in lever pressing in drug compared with saline pre-exposed rats. Together, these results demonstrate a direct relation between drug-induced sensitization of midbrain DA neuron reactivity and the excessive pursuit and self-administration of amphetamine.

The present results confirm previous reports of the effects of intraperitoneal amphetamine pre-exposure on self-administration of the drug using a PR schedule of reinforcement (Mendrek et al., 1998; Lorrain et al., 2000), establish that the same effects are produced by VTA, but not NAcc, amphetamine pre-exposure (experiment 1), and demonstrate that the enhanced drug selfadministration produced is tightly coupled to an enhancement in mesoaccumbens DA neuron reactivity (experiments 2 and 3). Importantly, manipulations known to block the induction of loco-
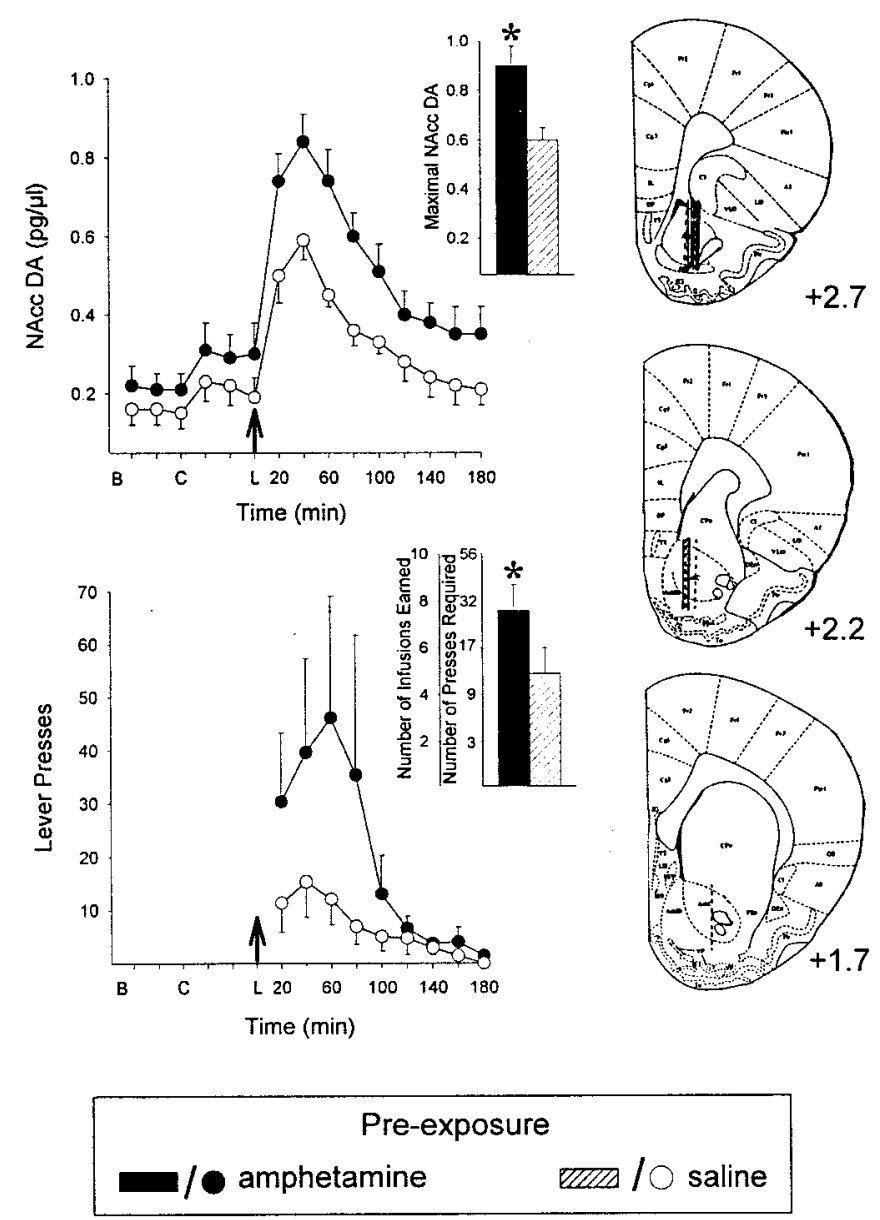

Figure 4. Rats previously exposed to amphetamine show enhanced NAcc DA overflow and lever pressing in response to a single priming injection of the drug. Top, Extracellular concentrations of DA in the NAcc before and after a single noncontingent priming injection of amphetamine $(1.0 \mathrm{mg} / \mathrm{kg}$, i.p.) on the fourth to the fifth day of PR testing. Data are shown as mean picograms per microliter $( \pm$ SEM). The priming injection was made as the levers were introduced into the chambers (arrow). Each scheduled completion had no intravenous consequences. Inset shows data as mean (+SEM) maximal NAcc DA overflow induced by amphetamine. ${ }^{*} p<0.005$ as revealed by $t$ test. Bottom, Nonreinforced lever presses (mean \pm SEM) after the priming injection (arrow) in the rats described above. Inset shows the mean (+SEM) number of infusions earned (not obtained) with the corresponding number of presses required. ${ }^{*} p<0.05$ as revealed by $t$ test. $B$, Baseline. $C$, Rats transferred to self-administration chamber. $L$, Lever inserted into chamber. Line drawings of coronal sections to the side show the location of the active portion of the microdialysis probes in the NAcc. Numbers indicate millimeters from bregma. Solid lines, Amphetamine pre-exposed; dashed lines, saline pre-exposed. $n=8$ per group.

motor and NAcc DA sensitization by amphetamine, such as administering a $D_{1}$ DA receptor antagonist before each drug preexposure injection (Bjijou et al., 1996; Vezina, 1996), have also been shown to block the enhancement in drug self-administration normally observed after systemic drug pre-exposure (Pierre and Vezina, 1998). Collectively, these findings strongly suggest that facilitation of amphetamine self-administration is a manifestation of sensitization to amphetamine that is mediated by the same neuronal mechanisms that underlie enhanced locomotion and dopamine overflow in response to the drug (Pierre and Vezina, 1998; Deroche et al., 1999). These neuronal mechanisms, initiated by antecedent pharmacological (Kalivas and Stewart, 1991) as well as 
nonpharmacological events (Piazza and Le Moal, 1996), may thus underlie the propensity of individuals to engage in drug-taking behavior as well as their liability for relapse, even after prolonged abstinence (De Vries et al., 1998; Tran-Nguyen et al., 1998; Grimm et al., 2001).

Just as amphetamine acts in the VTA, but not in the NAcc, to bring about sensitization of its effects on locomotor activity and DA release in the NAcc (Kalivas and Weber, 1988; Kalivas and Stewart, 1991; Hooks et al., 1992; Perugini and Vezina, 1994; Cador et al., 1995; Vezina, 1996), it appears able by its actions in this site to induce enduring changes that subsequently promote its pursuit and self-administration. These changes appear to be linked not to the act of drug taking, per se, because saline pre-exposed control rats will self-administer drug when little work is required and sufficiently high doses are available, as found with the FR1 and FR2 schedules in experiment 1 and as reported previously (Mendrek et al., 1998; Vezina et al., 1999; Lorrain et al., 2000). Rather they appear to be linked to enhanced motivation to engage in drug-taking behaviors that becomes particularly evident as the effort required to obtain the drug increases (Mendrek et al., 1998; Vezina et al., 1999; Lorrain et al., 2000; this study) or when low drug doses are accessible with little work (e.g., FR1 schedule; Piazza et al., 1989, 1990; Horger et al., 1990, 1992; Valadez and Schenk, 1994; Pierre and Vezina, 1997, 1998; Vezina et al., 1999). The resulting increase in drug taking may thus constitute a troubling behavioral expression of sensitization of the incentive motivational properties of the drug, a phenomenon that may be closely linked to drug craving and abuse in humans (Stewart et al., 1984; Robinson and Berridge, 1993; Deroche et al., 1999).

Enhanced mesoaccumbens DA neuron reactivity was manifested in the present experiments as a greater NAcc DA response to a noncontingent infusion of amphetamine and, when animals self-administered the drug, as an ability of these neurons to more readily maintain a NAcc DA response to amphetamine under conditions of progressively increasing workload. Although the precise mechanisms underlying these manifestations of enhanced neuronal reactivity remain elusive, there are several intercellular and intracellular pathways in place that could easily mediate these effects. For example, blockade of L- or N-type calcium channels and the inhibition of calcium-calmodulin-dependent kinase II has been shown to prevent the enhanced drug-induced DA release normally observed in the NAcc and striatum of stimulant sensitized rats (Pierce and Kalivas, 1997; Kantor et al., 1999). The release of DA by amphetamine has also been shown to require the activation of protein kinase C (Browman et al., 1998; Kantor and Gnegy, 1998). Various afferent inputs to the VTA and the NAcc using different neurotransmitters are well positioned to modulate the enhanced release of DA by stimulant drugs in sensitized animals by means of their actions on such intracellular pathways. These might include the voltage gating of calcium entry into DA neuron terminals by GABAergic and opioid afferent inputs (Morgan and Dewey, 1998; Gray et al., 1999) or actions at receptors with access to these and other kinases as with group I metabotropic glutamate receptors and protein kinase $\mathrm{C}$ (Pin and Duvoisin, 1995). It is likely, therefore, that the accrual of adaptations involving second messenger pathways in the mesoaccumbens DA neurons of amphetamine pre-exposed animals underlies, at least in part, the greater NAcc DA response observed in these sensitized animals after a noncontingent infusion of the drug (experiment 3) (Fig. 4). Such adaptations may also underlie the ability of these neurons to resist the progressive decrease in
NAcc DA responding imposed during amphetamine selfadministration by a progressively increasing workload. The effects of this progressively increasing workload could potentially be mediated by afferent inputs capable of regulating intracellular pathways like those described above (experiment 2) (Fig. 2C).

Rats for which the work required to obtain infusions was kept constant continued to press for the drug, and significant increases in NAcc DA overflow were observed in response to amphetamine through to the last infusion of the session. The decrease in the ability of amphetamine to maintain these effects under the increasingly demanding conditions of the PR schedule cannot therefore be accounted for by transmitter fatigue brought on by repeated drug exposure. Rather, it appears related to the increasing amount of work required to obtain successive inf usions. NAcc DA thus appears to encode the perceived value of the drug effect in relation to the work required to obtain it (Salamone et al., 1997) and to provide the motivational energy to continue responding (Stewart et al., 1984; Szechtman et al., 1994). This information may consequently determine how much responding will be emitted and whether it will continue to be emitted, both in animals self-administering the drug and those responding to a priming injection (Stewart et al., 1984; Marinelli et al., 1998; Stewart, 2000). By generating exaggerated responses to amphetamine, sensitized midbrain DA neurons thus may promote excessive drug seeking and taking whether in the drug-naive individual initiating the behavior when drug concentrations and cost are low (Piazza et al., 1989, 1990; Horger et al., 1990, 1992; Valadez and Schenk, 1994; Pierre and Vezina, 1997, 1998; Vezina et al., 1999) or in the drug-experienced individual self-administering substantially more drug under conditions of escalating cost.

Determining how sensitization of midbrain DA neuron reactivity develops and how its expression can be modulated may help elucidate how such behaviors characteristic of drug craving and abuse develop. It may also help decipher the mechanisms underlying individual differences in vulnerability to substance abuse. Animals that show a greater locomotor response to a novel environment have been reported to be more predisposed to engage in drug self-administration behaviors (Piazza et al., 1989, 1991; Piazza and Le Moal, 1996; Pierre and Vezina, 1997, 1998; Marinelli and White, 2000). Like psychomotor stimulant preexposed rats, these animals also show enhanced NAcc DA responding to novelty (Piazza et al., 1991), tail-pinch stress (RougePont et al., 1998), amphetamine (Bradberry et al., 1991), and cocaine (Hooks et al., 1991) as well as greater locomotor responding to NAcc infusions of DA (Hooks et al., 1994) and VTA DA cells that exhibit elevated firing rates and bursting activity (Marinelli and White, 2000).

\section{REFERENCES}

Bjijou Y, Stinus L, Le Moal M, Cador M (1996) Evidence for selective involvement of dopamine $\mathrm{D}_{1}$ receptors of the ventral tegmental area in the behavioral sensitization induced by intra-ventral tegmental area injections of D-amphetamine. J Pharmacol Exp Ther 277:1177-1187.

Bradberry CW, Gruen RJ, Berridge CW, Roth RH (1991) Individual differences in behavioral measures: correlations with nucleus accumbens dopamine measured by microdialysis. Pharmacol Biochem Behav 39:887-892

Browman KE, Kantor L, Richardson S, Badiani A, Robinson TE, Gnegy ME (1998) Injection of the protein kinase C inhibitor Ro31-8220 into the nucleus accumbens attenuates the acute response to amphetamine: tissue and behavioral studies. Brain Res 814:112-119.

Cador M, Bjijou Y, Stinus L (1995) Evidence of a complete independence of the neurobiological substrates for the induction and expression of behavioral sensitization to amphetamine. Neuroscience 65:385-395.

Crombag HS, Mueller H, Browman KE, Badiani A, Robinson TE (1999) A comparison of two behavioral measures of psychomotor activation 
following intravenous amphetamine or cocaine: dose- and sensitization-dependent changes. Behav Pharmacol 10:205-213.

Deroche V, Le Moal M, Piazza PV (1999) Cocaine self-administration increases the incentive motivational properties of the drug in rats. Eur J Neurosci 11:2731-2736.

De Vries T, Schoffelmeer ANM, Binnekade R, Mulder AH, Vanderschuren LJMJ (1998) Drug-induced reinstatement of heroin- and cocaine-seeking behavior following long-term extinction is associated with expression of behavioural sensitization. Eur J Neurosci 10:3565-3571.

Gray AM, Rawls SM, Shippenberg TS, McGinty JF (1999) The kappaopioid agonist, U-69593, decreases acute amphetamine-evoked behaviors and calcium-dependent dialysate levels of dopamine and glutamate in the ventral striatum. J Neurochem 73:1066-1074.

Grimm JW, Hope BT, Wise RA, Shaham Y (2001) Incubation of cocaine craving after withdrawal. Nature 412:141-142.

Hooks MS, Jones GH, Smith AD, Neill DB, Justice Jr JB (1991) Response to novelty predicts the locomotor and nucleus accumbens dopamine response to cocaine. Synapse 9:121-128.

Hooks MS, Jones GH, Liem BJ, Justice Jr JB (1992) Sensitization and individual differences to intraperitoneal amphetamine, cocaine or caffeine following repeated intracranial amphetamine infusions. Pharmacol Biochem Behav 43:815-823.

Hooks MS, Juncos JL, Justice Jr JB, Meiergerd SM, Povlock SL, Schenk JO, Kalivas PW (1994) Individual locomotor response to novelty predicts selective alterations in $\mathrm{D}_{1}$ and $\mathrm{D}_{2}$ receptors and mRNAs. J Neurosci 14:6144-6152.

Horger BA, Shelton K, Schenk S (1990) Pre-exposure sensitizes rats to the rewarding effects of cocaine. Pharmacol Biochem Behav 37:707-711.

Horger BA, Giles MK, Schenk S (1992) Pre-exposure to amphetamine and nicotine predisposes rats to self-administer a low dose of cocaine. Psychopharmacology 107:271-276.

Hull EM, Du J, Lorrain DS, Matuszewich L (1995) Extracellular dopamine in the medial preoptic area: implications for sexual motivation and hormonal control of copulation. J Neurosci 15:7465-7471.

Jolly D, Vezina P (1996) In vivo microdialysis in the rat: low cost and low labor construction of a small diameter, removable, concentric-style microdialysis probe system. J Neurosci Methods 68:259-267.

Kalivas PW, Stewart J (1991) Dopamine transmission in the initiation and expression of drug and stress-induced sensitization of motor activity. Brain Res Rev 16:223-244.

Kalivas PW, Weber B (1988) Amphetamine injected into the A10 dopamine region sensitizes rats to peripheral amphetamine and cocaine. J Pharmacol Exp Ther 245:1095-1102.

Kantor L, Gnegy ME (1998) Protein kinase C inhibitors block amphetamine-mediated dopamine release in rat striatal slices. J Pharmacol Exp Ther 284:592-598.

Kantor L, Keikilani Hewlett GH, Gnegy ME (1999) Enhanced amphetamine- and $\mathrm{K}^{+}$-mediated dopamine release in rat striatum after repeated amphetamine: differential requirements for $\mathrm{Ca}^{2+}$ and calmodulin-dependent phosphorylation and synaptic vesicles. J Neurosci 19:3801-3808.

Kirk RE (1968) Experimental design: procedures for the behavioral sciences. Pacific Grove, CA: Brooks/Cole.

Koob GF (1992) Drugs of abuse: anatomy, pharmacology and function of reward pathways. Trends Pharmacol Sci 13:177-184.

Lett BT (1989) Repeated exposures intensify rather than diminish the rewarding effects of amphetamine, morphine, and cocaine. Psychopharmacology 98:357-362.

Lorrain DS, Arnold GM, Vezina P (2000) Previous exposure to amphetamine increases incentive to obtain the drug: long-lasting effects revealed by the progressive ratio schedule. Behav Brain Res 107:9-19.

Marinelli M, White FJ (2000) Enhanced vulnerability to cocaine selfadministration is associated with elevated impulse activity of midbrain dopamine neurons. J Neurosci 20:8876-8885.

Marinelli M, Le Moal M, Piazza PV (1998) Sensitization to the motor effects of contingent infusions of heroin but not of $\kappa$ agonist RU 51599. Psychopharmacology 139:281-285.

Mendrek A, Blaha CD, Phillips AG (1998) Pre-exposure of rats to amphetamine sensitizes self-administration of this drug under a progressive ratio schedule. Psychopharmacology 135:416-422.

Morgan AE, Dewey SL (1998) Effects of pharmacologic increases in brain GABA levels on cocaine-induced changes in extracellular dopamine. Synapse 28:60-65.

Paxinos G, Watson C (1986) The rat brain in stereotaxic coordinates. New York: Academic.
Perugini M, Vezina P (1994) Amphetamine administered to the ventral tegmental area sensitizes rats to the locomotor effects of nucleus accumbens amphetamine. J Pharmacol Exp Ther 270:690-696.

Piazza PV, Le Moal M (1996) Pathophysiological basis for vulnerability to drug abuse: role of an interaction between stress, glucocorticoids and dopaminergic neurons. Annu Rev Toxicol 36:359-378.

Piazza PV, Deminiere JM, Le Moal M, Simon H (1989) Factors that predict individual vulnerability to amphetamine self-administration. Science 245:1511-1513.

Piazza PV, Deminiere JM, Le Moal M, Simon H (1990) Stress- and pharmacologically-induced behavioral sensitization increases vulnerability to acquisition of amphetamine self-administration. Brain Res 514:22-26.

Piazza PV, Rouge-Pont F, Deminiere JM, Kharouby M, Le Moal M, Simon H (1991) Dopaminergic activity is reduced in the prefrontal cortex and increased in the nucleus accumbens of rats predisposed to develop amphetamine self-administration. Brain Res 567:169-174.

Pierce RC, Kalivas PW (1997) Repeated cocaine modifies the mechanism by which amphetamine releases dopamine. J Neurosci 17:3254-3261.

Pierre PJ, Vezina P (1997) Predisposition to self-administer amphetamine: the contribution of response to novelty and prior exposure to the drug. Psychopharmacology 129:277-284.

Pierre PJ, Vezina P (1998) $\mathrm{D}_{1}$ dopamine receptor blockade prevents facilitation of amphetamine self-administration induced by prior exposure to the drug. Psychopharmacology 138:159-166.

Pin JP, Duvoisin R (1995) The metabotropic glutamate receptors: structure and functions. Neuropharmacology 34:1-26.

Ranaldi R, Pocock D, Zereik R, Wise RA (1999) Dopamine fluctuations in the nucleus accumbens during maintenance, extinction, and reinstatement of intravenous D-amphetamine self-administration. J Neurosci 19:4102-4109.

Richardson NR, Roberts DCS (1996) Progressive ratio schedules in drug self-administration studies in rats: a method to evaluate reinforcing efficacy. J Neurosci Methods 66:1-11.

Robinson TE, Berridge KC (1993) The neural basis of drug craving: an incentive-motivational theory of addiction. Brain Res Rev 18:247-291.

Rouge-Pont F, Deroche V, Le Moal M, Piazza PV (1998) Individual differences in stress-induced dopamine release in the nucleus accumbens are influenced by corticosterone. Eur J Neurosci 10:3903-3907.

Salamone JD, Cousins MS, Snyder BJ (1997) Behavioral functions of nucleus accumbens dopamine: empirical and conceptual problems with the anhedonia hypothesis. Neurosci Biobehav Rev 21:341-359.

Shaham Y, Erb S, Stewart J (2000) Stress-induced relapse to heroin and cocaine seeking in rats: a review. Brain Res Rev 33:13-33.

Shippenberg TS, Heidbreder CH (1995) Sensitization to the conditioned rewarding effects of cocaine: pharmacological and temporal characteristics. J Pharmacol Exp Ther 273:808-815.

Stewart J (2000) Pathways to relapse: the neurobiology of drug- and stress-induced relapse to drug taking. J Psychiat Neurosci 25:125-136.

Stewart J, Vezina P (1987) Environment-specific enhancement of the hyperactivity induced by systemic or intra-VTA morphine injections in rats pre-exposed to amphetamine. Psychobiology 15:144-153.

Stewart J, deWit H, Eikelboom R (1984) Role of unconditioned and conditioned drug effects in the self-administration of opiates and stimulants. Psychol Rev 91:251-268.

Szechtman H, Talangbayan H, Canaran G, Dai H, Eilam D (1994) Dynamics of behavioral sensitization induced by the dopamine agonist quinpirole and a proposed central energy control mechanism. Psychopharmacology 115:95-104.

Tran-Nguyen LTL, Fuchs RA, Coffey GP, Baker DA, O’Dell LE, Neisewander JL (1998) Time-dependent changes in cocaine-seeking behavior and extracellular dopamine levels in the amygdala during cocaine withdrawal. Neuropsychopharmacology 19:48-59.

Valadez A, Schenk S (1994) Persistence of the ability of amphetamine pre-exposure to facilitate acquisition of cocaine self-administration. Pharmacol Biochem Behav 47:203-205.

Vezina $\mathrm{P}$ (1996) $\mathrm{D}_{1}$ dopamine receptor activation is necessary for the induction of sensitization by amphetamine in the ventral tegmental area. J Neurosci 16:2411-2420.

Vezina P, Pierre PJ, Lorrain DS (1999) The effect of previous exposure to amphetamine on drug-induced locomotion and self-administration of a low dose of the drug. Psychopharmacology 147:125-134.

Wise RA, Bozarth MA (1987) A psychomotor stimulant theory of addiction. Psychol Rev 94:469-492. 